\title{
Chest Wall Motion after Thixotropy Conditioning of Inspiratory Muscles in Healthy Humans
}

\author{
Masahiko IZUMIZAKI, Yasuyoshi OHSHIMA, Michiko IWASE, and Ikuo HOMMA \\ Department of Physiology, Showa University School of Medicine, 1-5-8 Hatanodai, Shinagawa-ku, Tokyo, 142-8555 Japan
}

\begin{abstract}
Inspiratory muscle conditioning at a lower or higher lung volume based on the principles of muscle thixotropy causes acute changes in end-expiratory chest wall and lung volumes. The present study aimed to demonstrate the time course of effects of this conditioning on both end-expiratory chest wall volume and thoracoabdominal synchrony. We measured chest wall motion with respiratory induction plethysmography at $0.5,1,2$, 3 , and 6 min after conditioning at three different lung volumes in 15 healthy men. After conditioning at total lung capacity $-20 \%$ inspiratory capacity, increases in end-expiratory chest wall volume were significant at $0.5,1$, and $2 \mathrm{~min}(P<0.05)$, being most obvious at $0.5 \mathrm{~min}(\Delta 0.24 \pm 0.20$ liter). After conditioning at residual volume, reductions in end-expiratory chest wall volume
\end{abstract}

were significant at any time point $(P<0.05)$, being most obvious at $0.5 \mathrm{~min}(\Delta 0.16 \pm 0.08$ liter). Conditioning at functional residual capacity had little effect on the volume. Spirometric inspiratory capacity at 6 min after conditioning at residual volume (2.68 \pm 0.35 liter) was higher than the baseline value $(2.53 \pm 0.31$ liter, $P$ $<0.05)$. Reductions in the phase angle, quantified by the KonnoMead diagram, occurred after conditioning at residual volume at any time point $(P<0.05)$, being most obvious at $2 \min (\Delta 3.47 \pm$ 3.02 degrees). In conclusion, there is a 6 -min time course of changes in end-expiratory chest wall volume after conditioning. More synchronous motion between the rib cage and abdomen occurs after conditioning at residual volume.

Key words: chest wall, inspiratory muscles, rib cage, thixotropy, thoracoabdominal synchrony.

$\mathrm{S}_{\mathrm{k} e \mathrm{a}}$ that makes stiffness and resting tension at a given muscle that makes stiffness and resting tension at a given muscle length dependent on the previous history of movements and contractions [1-4]. This property, thixotropy, is affected by the status of cross-bridges between actin and myosin filaments [5]. A particularly prominent manifestation of muscle thixotropy is that the passive stiffness of a muscle depends on whether the muscle was contracted immediately beforehand at a long length (hold-long conditioning) or at a short length (hold-short conditioning) [1, 4]. This process increases stiffness of the muscle under the hold-short conditioning, but it decreases stiffness of the muscle under the hold-long conditioning.

We have reported that inspiratory muscle conditioning based on the principles of muscle thixotropy acutely changes end-expiratory chest wall volume (Vcw) and lung volume in healthy humans and patients with chronic obstructive pulmonary disease (COPD) [6-10]. Thixotropy conditioning of inspiratory muscles involves inspiratory muscle contraction at an inflated or deflated lung volume [6-10]. Inspiratory muscles include the diaphragm, the external intercostals, and the parasternal intercostals, and these muscles shorten and lengthen with lung inflation and deflation, respectively [11-14]. Conditioning at an inflated lung volume, which corresponds to hold-short conditioning to increase muscle stiffness, is followed by an increase in end-expiratory $\mathrm{Vcw}$, measured with respiratory induction plethysmography (RIP), and conditioning at a deflated lung volume, which corresponds to hold-long conditioning to decrease muscle stiffness, is followed by a reduction in end-expiratory $\mathrm{Vcw}$ [7].

The time course of chest wall motion after thixotropy conditioning of inspiratory muscles has not been identified. Our previous reports have highlighted the effects of muscle thixotropy on end-expiratory volumes during several breath cycles, although changes in spirometrically determined inspiratory capacity (IC) after thixotropy conditioning, which may mirror end-expiratory lung volume, extend over a period of $1 \mathrm{~min}$ [7]. The time course of muscle thixotropy has been shown in limb muscles. Muscle spindle responsiveness of a toad muscle to a stretch remained reduced for 5 min after hold-long [15]. Changes in the stiffness of human limb muscles induced by thixotropy conditioning last for several to $10 \mathrm{~min}$ [2]. These findings suggest that the effects of thixotropy conditioning of inspiratory muscles on chest wall motion would last over a period of minutes.

The coordination of chest wall motion has been investigated particularly in the context of respiratory diseases. During the breathing of healthy humans, the rib cage and 
abdominal compartments increase and decrease in synchrony. However, thoracoabdominal asynchrony is common in patients with COPD, in particular in those having lung hyperinflation $[16,17]$. Nevertheless, it remains unclear whether thixotropic inflation/deflation would change the coordination of abdominal and rib cage motions.

The present study aimed to demonstrate the time course of the effects of thixotropy conditioning of inspiratory muscles on end-expiratory $\mathrm{Vcw}$ and the coordination of abdominal and rib cage motions. We characterized the values of RIP measurements including a thoracoabdominal asynchrony index, the phase angle, quantified by the Konno-Mead diagram. Our hypotheses were first, that thixotropic inflation/deflation of the chest wall lasts for several minutes, and second, that thixotropy conditioning affects the coordination of the abdominal and rib cage motions.

\section{METHODS}

Subjects. The subjects were 15 healthy men (age range, 20-29 years) with no history of chronic pulmonary or neuromuscular disease. This study was approved by the Ethics Committee of Showa University and was performed in accordance with the Declaration of Helsinki. All subjects provided written informed consent for participation.

Apparatus. Each subject was seated on a chair during the trials with his nose clipped, and he wore a mouthpiece to which a transducer (AR-601G; Nihon Kohden, Tokyo, Japan) was attached to measure mouth pressure. A magnetically driven mouthpiece shutter was triggered manually to close the airway. We measured chest wall movement with RIP (the LifeShirt system; VivoMetrics, Ventura, USA) and lung volume with a respiratory flowmeter (RF-2; Minato Medical, Osaka, Japan). The LifeShirt system consists of the LifeShirt garment, LifeShirt recorder, and VivoLogic software [18]. RIP sensors consist of sinusoidal arrays of electrical wires and are woven into the garment. The system monitors chest wall motion by passing a continuous, low voltage electrical current through the two sensors that surround the rib cage and abdomen. RIP was calibrated by the least squares method, using sitting and standing postures with rebreathing into a fixed bag volume ( 0.8 liter). Data from RIP were fed into a LifeShirt Recorder and analyzed with VivoLogic software. Data from the flowmeter and transducer were fed into a computerized analysis system (PowerLab; ADInstruments, Castle Hill, Australia).

Prior to the experiment, IC and expiratory reserve volume (ERV) were estimated with the flowmeter at least three times to determine target lung volumes at which each conditioning maneuver should be done. IC was measured when the subject took a slow full inspiration with no
A

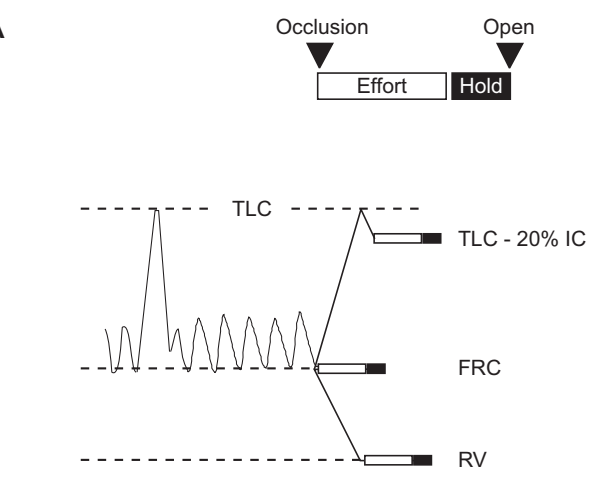

B
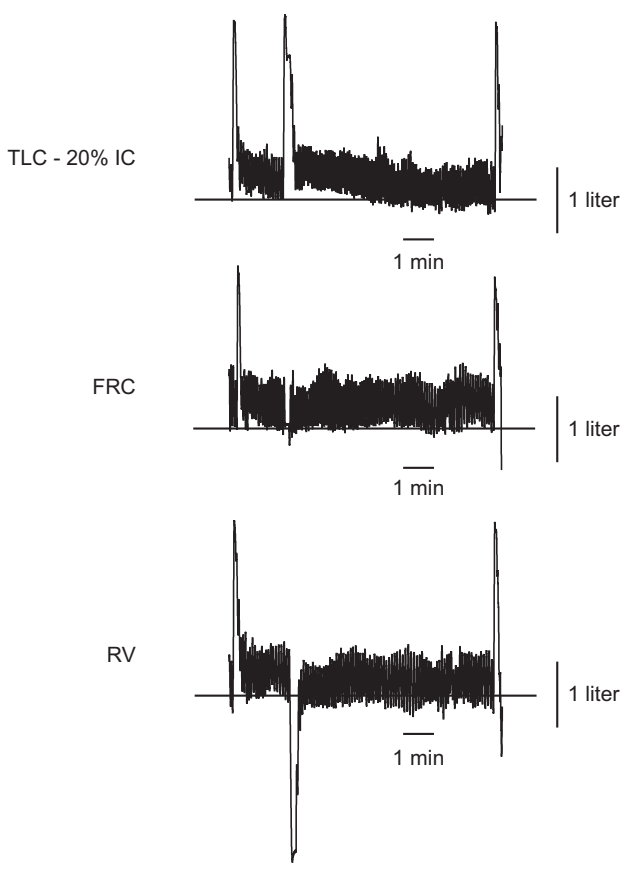

Fig. 1. Schematic representation of conditioning and examples of chest wall motion before and following conditioning. (A) Inspiratory muscle conditioning consisted of a 5-s inspiratory effort at $30 \%$ of maximal inspiratory mouth pressure (open squares) and a subsequent breath-holding period for 2-3 s (solid squares) at one of the three lung volumes [total lung capacity (TLC) $-20 \%$ inspiratory capacity (IC), functional residual capacity (FRC), or residual volume (RV)] with airway occlusion. After each conditioning maneuver, the airway was reopened and the subject resumed quiet breathing for 6 min. Conditioning was preceded by a TLC maneuver. (B) Examples of a chest wall volume ( $\mathrm{Vcw}$ ) trace before and following three types of inspiratory muscle conditioning performed by a single subject. Conditioning at TLC $-20 \%$ IC is followed by increases in end-expiratory Vcw (top). These increases are maintained approximately for $2 \mathrm{~min}$. Conditioning at FRC produces few changes in end-expiratory $\mathrm{Vcw}$, with the exception of the next several breath cycles (middle). Conditioning at RV produces obvious reductions in end-expiratory Vcw (bottom). These reductions are maintained until the end of the test. The TLC maneuver to measure IC was performed before and at the end of each test, and differences in the TLC position are small. Upward deflections of traces correspond to inspiratory movements. 
hesitation from a position of end-tidal expiration. ERV was measured when the subject took a slow full expiration from a position of end-tidal expiration. Each measurement was performed $1 \mathrm{~min}$ after an inspiratory maneuver to a total lung capacity (TLC maneuver) to standardize volume history. Maximal inspiratory mouth pressures (MIP) at three lung volume levels (TLC $-20 \%$ IC, functional residual capacity $[\mathrm{FRC}]$, and residual volume $[\mathrm{RV}]$ ) were also determined. Lung volume of TLC $-20 \%$ IC was determined by exhaling gas volume of $20 \%$ IC from the TLC position.

Experimental protocol. The protocol included three different types of thixotropy conditioning maneuvers, shown schematically in Fig. 1A. The subject was instructed to make an inspiratory effort at $30 \%$ MIP at one of the three levels of lung volume (TLC $-20 \%$ IC FRC, or RV) in random order. The effort was maintained for $5 \mathrm{~s}$ with a subsequent breath-holding period for 2-3 s with airway occlusion. After each conditioning maneuver, the shutter was reopened, and the subject resumed breathing. Each test was performed at least twice.

During the trials, lung volume signals were monitored online to determine when the airway was closed. A readout of mouth pressure enabled the subject to adjust the pressure to the target level during conditioning. The trials were separated by rest periods of at least $3 \mathrm{~min}$. The TLC maneuver to measure IC was performed twice: $1 \mathrm{~min}$ before each test and at the end of each test, and both ICs were compared. We also calculated differences in the TLC position of the Vcw trace, which were standardized by IC measured at the end of each test. All volume measurements by RIP were corrected by the flowmeter, using the ratio of IC by RIP over IC.

For quantitative analysis, the mean end-expiratory volume of $30 \mathrm{~s}$ taken immediately before each conditioning was defined as the zero level for each RIP trace. Differences in end-expiratory volume between the zero line and after each conditioning were measured in each RIP trace. The phase angle was used to determine thoracoabdominal synchrony, which was calculated using the VivoLogic software according to a Konno-Mead Lissajous loop [19, 20]. Asynchronous motion widens the loop; the phase angle comprised between $0^{\circ}$ (complete synchrony) and $180^{\circ}$ (complete asynchrony). Respiratory frequency (Rf, breaths $\mathrm{min}^{-1}$ ), expiratory tidal volume of the chest wall $\left(\mathrm{V}_{\mathrm{TE}} \mathrm{cw}\right.$, liter), minute ventilation of the chest wall $\left(\dot{\mathrm{V}}_{\mathrm{E}} \mathrm{cW}\right.$, liter $\left.\min ^{-1}\right)$, inspiratory time $\left(\mathrm{T}_{\mathrm{I}}, \mathrm{s}\right)$, and expiratory time $\left(T_{E}, s\right)$ were computed breath-by-breath by the VivoLogic software. The software also provided us with the thoracic contribution to the tidal volume, expressed as the ratio of the inspiratory tidal volume of the rib cage to the inspiratory tidal volume of the chest wall (\% RC). These variables were measured at post $0.5,1,2,3$, and $6 \mathrm{~min}$. A 30 -s average immediately before each time point was taken for each variable. Baseline measurements were taken for $30 \mathrm{~s}$ immediately before each conditioning maneuver.

Statistics. Results are expressed as means \pm SD. The time trend in end-expiratory volumes, the phase angle, breathing pattern parameters, $\% \mathrm{RC}$, and the TLC position of $\mathrm{Vcw}$ trace was analyzed by a one-way analysis of variance (ANOVA) for repeated measures followed by Dunnett's test, with the baseline value used as the reference value (InStat; GraphPad, San Diego, USA). Spirometrically determined IC was analyzed with paired $t$-test with Bonferroni correction for multiple comparisons (InStat). Significance was accepted at $P<0.05$. Sample size calculations for the present study were based on changes in the IC reported in a previous study [7], in which we observed means $\pm \mathrm{SD}$ for post IC at $1 \mathrm{~min}$ of $-0.13 \pm 0.12$ liter (conditioning at FRC $+60 \%$ IC) and $0.12 \pm 0.10$ liter (conditioning at RV), though the primary outcome of the present study was the change in end-expiratory $\mathrm{Vcw}$. We assumed an alpha level of 0.01 to account for multiple comparisons. The sample size of 12 subjects allowed $>80 \%$ statistical power to test within-conditioning comparison to distinguish differences from chance alone (StatMate; GraphPad).

\section{RESULTS}

\section{Subject characteristics}

Mean MIPs of the subjects were $64.0 \pm 35.1 \mathrm{~cm} \mathrm{H}_{2} \mathrm{O}$ (TLC $-20 \%$ IC), $126.1 \pm 38.3 \mathrm{~cm} \mathrm{H}_{2} \mathrm{O}$ (FRC), and 155.1 $\pm 51.6 \mathrm{~cm} \mathrm{H}_{2} \mathrm{O}(\mathrm{RV})$. Spirometrically determined IC and ERV before experiments were $2.63 \pm 0.32$ liter BTPS and $2.13 \pm 0.32$ liter BTPS, respectively.

\section{End-expiratory volumes and IC}

RIP measurements showed the effects of thixotropy conditioning of inspiratory muscles on chest wall movements. Changes in Vcw for breaths before and after the conditioning maneuvers performed by a subject are illustrated in Fig. 1B. In this figure, end-expiratory Vcw increases after conditioning at TLC $-20 \%$ IC, and the increase is maintained for about $2 \mathrm{~min}$. Conditioning at RV shifts end-expiratory Vcw below the baseline. This shift is maintained until the last TLC maneuver occurs.

The left panel of Fig. 2A shows the aftereffects of all types of conditioning on the time course of changes in end-expiratory $\mathrm{Vcw}(\Delta$ end-expiratory $\mathrm{Vcw})$. After conditioning at TLC $-20 \%$ IC, an increase in end-expiratory Vcw was significant at $0.5,1$, and 2 min (all $P<0.05$ ), which were most obvious at $0.5 \mathrm{~min}$ and then subsided. After conditioning at $\mathrm{RV}$, reductions in end-expiratory Vcw were significant at any time points (all $P<0.05$ ), which were most obvious at $0.5 \mathrm{~min}$. Conditioning at FRC had little effect on end-expiratory Vcw. Measurements of spirometrically determined IC were in agreement with $\Delta$ end-expiratory Vcw (Fig. 2B). The IC at 6 min after conditioning at RV was higher than the baseline value $(P<$ 
TLC - $20 \%$ IC

$\square$ FRC

$\mathrm{RV}$
Chest wall (Vcw)

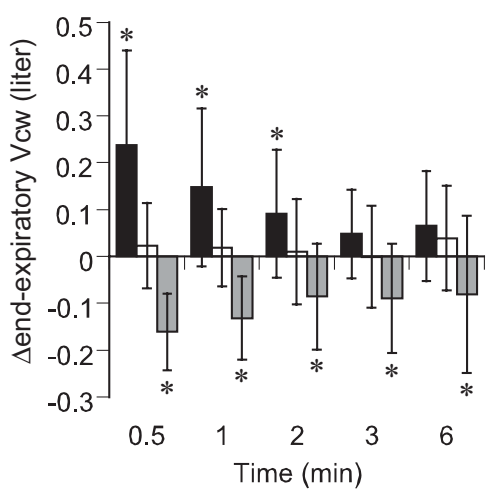

B

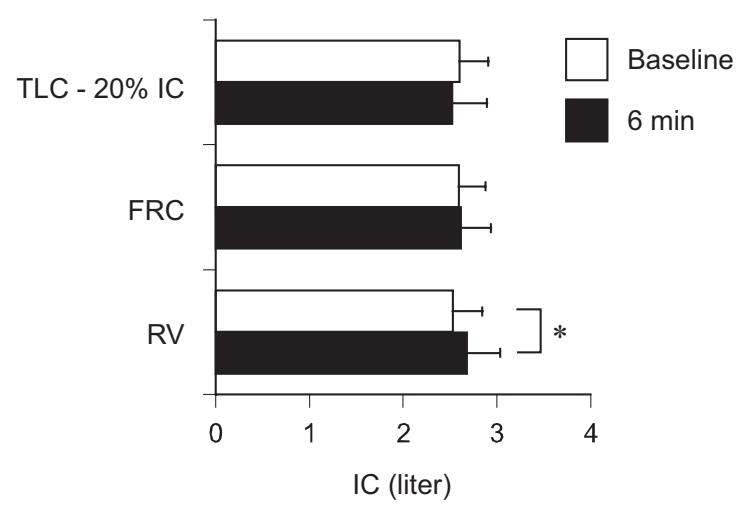

Fig. 2. End-expiratory volumes after conditioning over time. (A) Time course of changes in end-expiratory Vcw ( $\Delta$ end-expiratory $\mathrm{Vcw}$, left), rib cage volume ( $\Delta$ end-expiratory $\mathrm{Vrc}$, middle), and abdominal volume ( $\Delta$ end-expiratory $\mathrm{Vab}$, right) following conditioning at three different lung volumes (TLC - $20 \%$ IC, FRC, and RV). Chest wall inflation was maintained for 2 min on average after conditioning at TLC $-20 \%$ IC, and chest wall deflation was maintained for 6 min after conditioning at RV (left panel). After conditioning at TLC $-20 \%$ IC, a significant reduction in end-expiratory Vab was observed at 2, 3, and 6 min (right panel), where end-expiratory $\mathrm{Vrc}$ was still maintained above its baseline level (middle panel). As a result, this abdominal deflation minimized overall chest wall inflation resulting from the rib cage inflation. "Significant difference from the baseline value $(P<0.05)$. Values are means \pm SD for 15

0.05). Such a difference in the IC was not found after other conditionings. Mean differences in the TLC position of Vcw trace were less than $2 \%$ of IC and did not differ between conditionings (Fig. 2C), suggesting that artifactual changes in end-expiratory $\mathrm{Vcw}$ were minimized in the present study.

After conditioning at TLC $-20 \%$ IC, the time course of
C

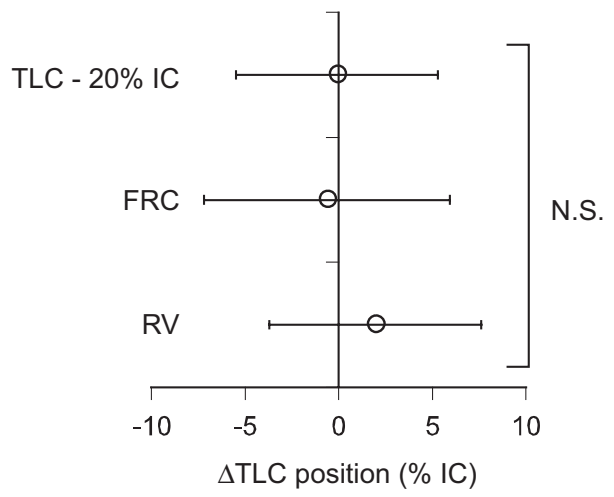

subjects. (B) Spirometrically determined IC was measured before (baseline) and after conditioning (6 min). The IC at $6 \mathrm{~min}$ after conditioning at RV was higher than the baseline value, which was in line with the changes in end-expiratory Vcw. "Significant difference between bars $(P<0.05)$. Values are means \pm SD for 15 subjects. (C) Differences in the TLC position ( $\triangle T L C$ position) of $\mathrm{Vcw}$ trace between before and after conditioning, which were standardized by IC measured at $6 \mathrm{~min}(\%$ IC). Mean differences in the TLC position of Vcw trace were less than $2 \%$ of IC and did not differ between conditionings, suggesting that artifactual alterations in end-expiratory Vcw were minimized. Positive values imply that the TLC position at $6 \mathrm{~min}$ is higher than the baseline value. Values are means \pm SD for 15 subjects.

$\Delta$ end-expiratory $\operatorname{Vrc}$ was similar, but not the same as that of $\Delta$ end-expiratory Vcw (Fig. 2A, middle panel). An increase in end-expiratory Vrc was maintained throughout the tests (all $P<0.05$ ), which accounted for the increase in end-expiratory Vcw. However, after this conditioning a significant reduction in end-expiratory Vab occurred at 2 , 3 , and $6 \mathrm{~min}$ (all $P<0.05)$ (Fig. $2 \mathrm{~A}$, right panel), where 
A
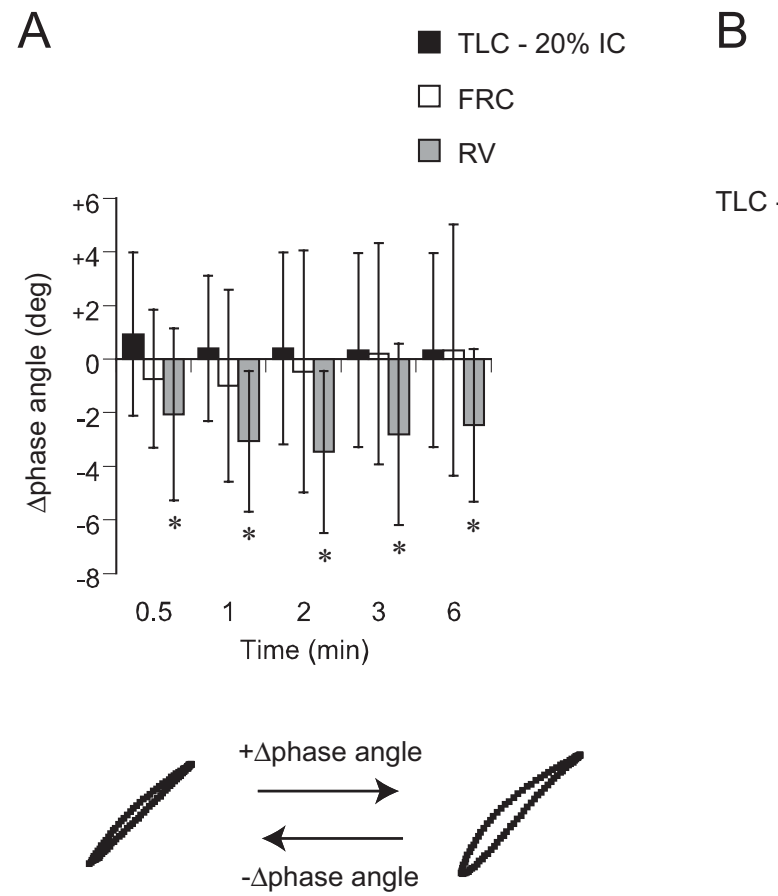

Fig. 3. Coordination of the abdominal and rib cage motion after conditioning. (A) Changes in the phase angle ( $\Delta$ phase angle) following conditioning at three different lung volumes (TLC $20 \%$ IC, FRC, and RV). Reductions in the phase angle occurred after conditioning at RV at any time points, indicating a more synchronous motion between the rib cage and abdo-

end-expiratory Vrc was still maintained above its baseline level.

After conditioning at RV, the partitioning of thixotropic deflation in the chest wall compartments changed with time. A reduction in end-expiratory Vrc was significant only at 0.5 and $1 \mathrm{~min}$ (both $P<0.05$ ) (Fig. 2A, middle panel), which largely explained the reduction in end-expiratory $\mathrm{Vcw}$ at these time points. Thereafter the reduction in end-expiratory Vrc practically disappeared. On the other hand, end-expiratory Vab showed a significant reduction (all $P<0.05$ ), with the exception of at $2 \mathrm{~min}$ (Fig. 2A, right panel). Accordingly, the contribution of the abdominal compartment to the reduction in end-expiratory $\mathrm{Vcw}$ increased as time increased.

\section{Thoracoabdominal synchrony}

Changes in the phase angle ( $\Delta$ phase angle) of 30 -s average are shown in Fig. 3A. Mean values of the baseline phase angle were $7.1 \pm 2.5$ degrees (TLC $-20 \%$ IC), $7.7 \pm$ 3.1 degrees (FRC), and $8.9 \pm 2.9$ degrees (RV). Reductions in the phase angle occurred after conditioning at RV at any time points (all $P<0.05$ ). Representative KonnoMead loops from a single subject show a reduction in the width of the loop after conditioning at RV (Fig. 3B); each loop was obtained from one breath cycle. There was outward motion of the rib cage and abdomen during inspiration regardless of which conditioning preceded.

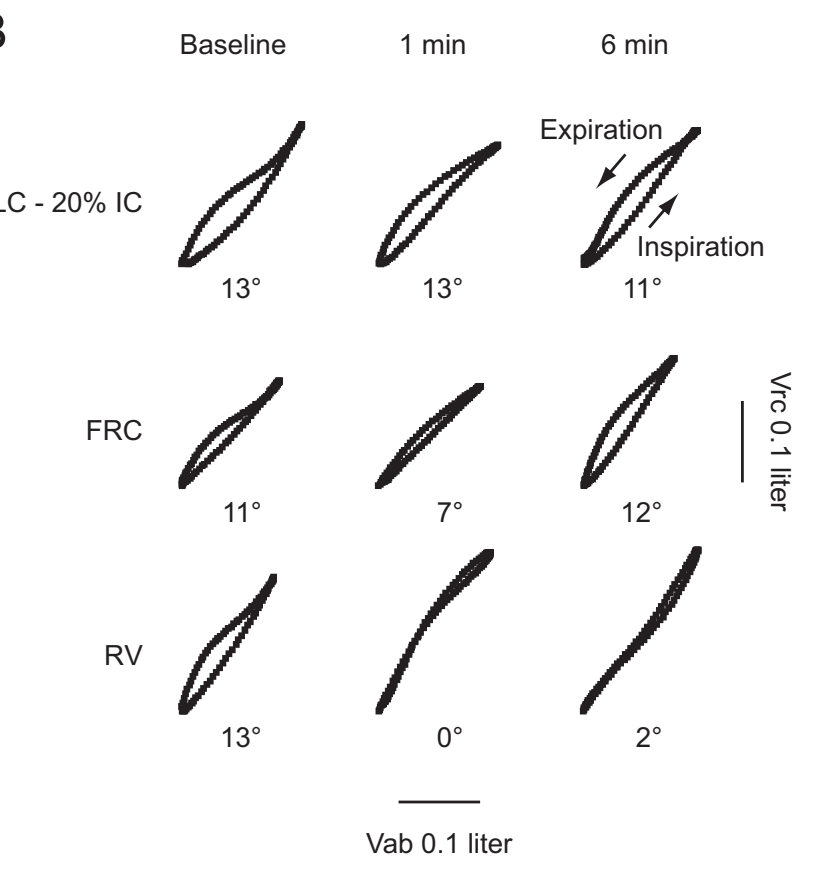

men. *Significant difference from the baseline value $(P<0.05)$. Values are means \pm SD for 15 subjects. (B) Examples of Konno-Mead Lissajous loops with calculated phase angles recorded in a subject. The width of the loop decreased after conditioning at RV.

\section{Breathing pattern and \% RC}

The aftereffects of conditioning on breathing patterns and $\% \mathrm{RC}$ are shown in Fig. 4. $\mathrm{V}_{\mathrm{TE}} \mathrm{cW}$ increased at 2, 3, and 6 min regardless of type of conditioning (all $P<0.05$ ). The $\%$ RC remained practically constant throughout the tests. One-way ANOVA did not detect significant changes in $\mathrm{T}_{\mathrm{I}}$ or $\mathrm{T}_{\mathrm{E}}$ (data not shown).

\section{DISCUSSION}

In the present study, we measured the chest wall motion with RIP after thixotropy conditioning of inspiratory muscles. We showed the time course of effects of this conditioning on end-expiratory Vcw. We also found a reduction in the phase angle, quantified by the Konno-Mead diagram, after thixotropy conditioning at RV, suggesting that the conditioning would affect thoracoabdominal synchrony.

We showed the time course of $\Delta$ end-expiratory $\mathrm{Vcw}$ after thixotropy conditioning. End-expiratory $\mathrm{Vcw}$ increased after conditioning at TLC $-20 \%$ IC. This thixotropic inflation of the chest wall was maintained for $2 \mathrm{~min}$ on average. In contrast, thixotropic deflation of the chest wall following conditioning at RV was still observed at 6 min. These findings were supported by another finding that spirometrically determined IC showed an increase at 6 min after conditioning at RV. In a previous paper, we 

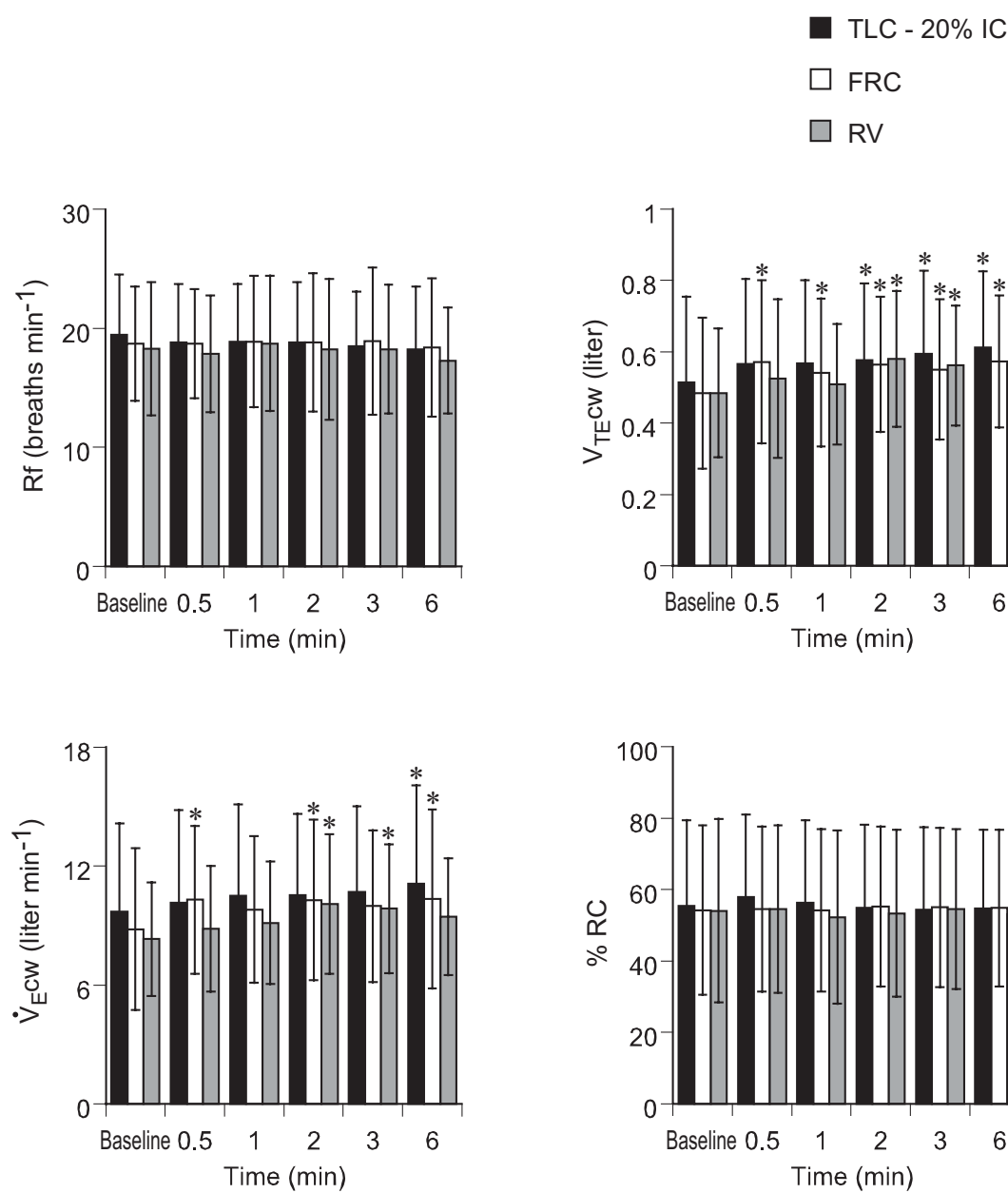
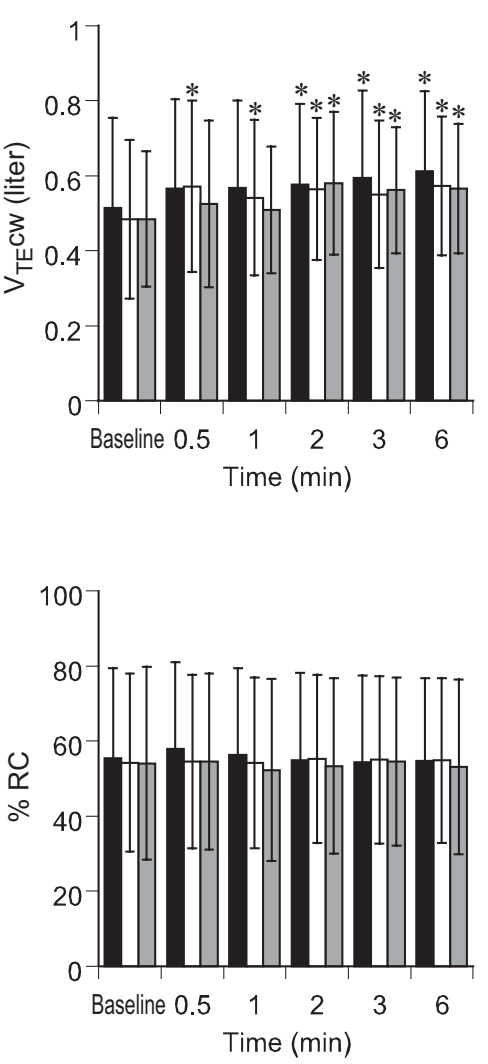

Fig. 4. Effects of thixotropy conditioning on breathing patterns. Time course of changes in respiratory frequency (Rf), expiratory tidal volume of the chest wall $\left(\mathrm{V}_{\mathrm{TE}} \mathrm{CW}\right)$, minute ventilation of the chest wall $\left(\dot{\mathrm{V}}_{\mathrm{E}} \mathrm{CW}\right)$, and the ratio of inspiratory tidal volume of the rib cage to that of the chest wall (\% RC) following conditioning at three different lung volumes (TLC $20 \% \mathrm{IC}, \mathrm{FRC}$, and RV). $\mathrm{V}_{\mathrm{TE}} \mathrm{CW}$ increased at 2 , 3 , and 6 min regardless of type of conditioning. *Significant difference from the baseline value $(P<0.05)$. Values are means \pm SD for 15 subjects. measured IC with a spirometer at $0.5,1$, and 3 min after thixotropy conditioning [7]. We observed a reduction in the IC at 0.5 and $1 \mathrm{~min}$ after conditioning at a higher lung volume (FRC $+60 \%$ IC); however, this reduction disappeared at $3 \mathrm{~min}$. That study also showed increased IC at $0.5,1$, and 3 min after conditioning at RV. These previous data are in line with the time course of $\Delta$ end-expiratory Vcw we found in the current study. The use of the RIP method raises a concern that some of the volume shifts in end-expiratory Vcw we found may be artifactual because movement of the garment or a change in body position would occur. Werchowski [21] showed that estimates of volume changes from measurements of cross-sectional areas of the chest wall introduce errors in measuring the change in FRC. However, there was a slight difference in the TLC position of the Vcw trace between before and at 6 min after conditioning. This indicated that $\Delta$ end-expiratory $\mathrm{Vcw}$ we found were valid, on the assumption that thixotropy conditioning does not change the volume of TLC.

Time course of $\Delta$ end-expiratory Vrc and $\Delta$ end-expiratory Vab differed from $\Delta$ end-expiratory Vcw. After conditioning at TLC $-20 \%$ IC, the rib cage component largely accounted for the volume of the chest wall inflation, in particular at $0.5,1$, and $2 \mathrm{~min}$, at which no inflation occurred in the abdominal compartment. These findings suggest that thixotropy of rib cage inspiratory muscles plays an important part in this chest wall inflation. However, this two-compartment model of the chest wall cannot exclude the possibility that thixotropy of the diaphragm contributes to thixotropic inflation of the chest wall because the diaphragm acts directly on the lower part of the rib cage [22].

End-expiratory Vab started to decrease at $2 \mathrm{~min}$ after this conditioning. As a result, this abdominal deflation minimized overall chest wall inflation resulting from the rib cage inflation. Accordingly, increases in end-expiratory $\mathrm{Vcw}$ disappeared at $3 \mathrm{~min}$, even though an increase in end-expiratory Vrc was maintained until the end of the test. The recruitment of expiratory abdominal muscles may explain this decrease in end-expiratory Vab after conditioning at TLC $-20 \%$ IC. Maintained inflation of the lung evokes abdominal muscle activity through pulmonary vagal receptors in cats [23]. Abdominal muscle recruitment also occurs with lung hyperinflation caused by continuous positive airway pressure in supine dogs [24]. In humans, chest wall hyperinflation caused by histamineinduced bronchoconstriction is accompanied by abdominal muscle recruitment in patients with asthma and COPD $[25,26]$. As a consequence, in those patients, the rib cage compartment largely accounts for the volume of the chest 
wall hyperinflation. The reduction in end-expiratory Vab seemed to preserve the length of the diaphragm at the start of inspiration and thereby prevent the force generation ability of the diaphragm from decreasing when thixotropic hyperinflation occurred.

We also found a small but significant reduction in the phase angle after conditioning at RV with deflation of the chest wall. Although we did not find other papers addressing whether experimental deflation of the chest wall changes thoracoabdominal synchrony, this finding suggests that chest wall deflation improves thoracoabdominal synchrony. Our speculation on a mechanism to explain this finding is that the deflation increases the area of apposition of the diaphragm to the rib cage and consequently improves the mechanical coupling between the rib cage and abdomen. Additionally, we speculate that increased stiffness of the abdominal wall induced by thixotropy may efficiently couple the rib cage and abdomen. Using upper limb muscles, Hagbarth et al. [2] showed that a forceful shortening contraction is followed by an enduring increase in stiffness of the muscle. Abdominal muscle contraction to exhale to RV could lead to a stiffness of the muscles and thus to a stiffness of the abdominal wall. Our speculations, however, await further experimental proof.

Use of the RIP method may raise another concern that the two-compartment chest wall model used is insufficient in estimating the coordination of the abdominal and rib cage motions [27]. Aliverti et al. [28] analyzed the chest wall motion during exercise in healthy humans based on the three-compartment chest wall model with optoelectronic plethysmography. This model would provide more information on changes in the coordination of the abdominal and rib cage motions after thixotropy conditioning.

The findings of the current study suggest that thixotropy conditioning is beneficial for patients with COPD. Lung hyperinflation is an important physiological abnormality in such patients. A reduction in elastic recoil of the lung increases resting lung volume statically in COPD, and lung hyperinflation progresses dynamically during exercise because of limited airflow, contributing to the occurrence of dyspnea and limiting exercise capacity [29, 30]. Thixotropy conditioning at RV performed prior to exercise may reduce hyperinflation during the exercise. However, the effect of the conditioning on dynamic lung hyperinflation needs to be further investigated before it can be recommended for clinical use because change in the pressure-volume relationship of the respiratory system is considered to play a minor role in the dynamic lung hyperinflation.

A limitation of the present work is that we did not clearly identify to what extent other factors were involved in chest wall inflation and deflation. Factors that could influence $\Delta$ end-expiratory volume include respiratory reflexes mediated by vagal afferents, which could activate abdominal muscles as discussed above, and those by chemical stimuli induced potentially by the instrumental dead space, which may explain a gradual increase in $\mathrm{V}_{\mathrm{TE}} \mathrm{cW}$ following conditioning. These factors may have modified the effects originating in the thixotropy mechanism on overall chest wall movements.

The present study demonstrates that there is a 6-min time course of the chest wall motion after thixotropy conditioning of inspiratory muscles. Thixotropy conditioning has effects on end-expiratory Vcw in the respiratory cycles that follow. We showed that there is a more synchronous motion between the rib cage and abdomen after thixotropy conditioning at RV.

This study was supported in part by a Grant-in-Aid for Scientific Research from the Ministry of Education, Culture, Sports, Science and Technology of Japan (Grant no. 17790542).

\section{REFERENCES}

1. Gregory JE, Wise AK, Wood SA, Prochazka A, Proske U. Muscle history, fusimotor activity and the human stretch reflex. J Physiol. 1998;513:927-34.

2. Hagbarth KE, Hagglund JV, Nordin M, Wallin EU. Thixotropic behaviour of human finger flexor muscles with accompanying changes in spindle and reflex responses to stretch. J Physiol. 1985;368:323-42.

3. Lakie M, Walsh EG, Wright GW. Resonance at the wrist demonstrated by the use of a torque motor: an instrumental analysis of muscle tone in man. J Physiol. 1984;353:265-85.

4. Proske U, Morgan DL, Gregory JE. Thixotropy in skeletal muscle and in muscle spindles: a review. Prog Neurobiol. 1993;41:705-21.

5. Whitehead NP, Gregory JE, Morgan DL, Proske U. Passive mechanical properties of the medial gastrocnemius muscle of the cat. J Physiol. 2001;536:893-903.

6. Homma I, Hagbarth KE. Thixotropy of rib cage respiratory muscles in normal subjects. J Appl Physiol. 2000;89:1753-8.

7. Izumizaki M, Iwase M, Ohshima Y, Homma I. Acute effects of thixotropy conditioning of inspiratory muscles on end-expiratory chest wall and lung volumes in normal humans. J Appl Physiol. 2006a;101:298-306.

8. Izumizaki M, Kakizaki F, Tanaka K, Homma I. Immediate effects of thixotropy conditioning of inspiratory muscles on chest-wall volume in chronic obstructive pulmonary disease. Respir Care. 2006b;51:750-7.

9. Izumizaki M, Shibata M, Homma I. Factors contributing to thixotropy of inspiratory muscles. Respir Physiol Neurobiol. 2004;140:257-64.

10. Shibata M, Izumizaki M, Homma I. The activation of muscle spindles enhances the thixotropic behavior of rib cage respiratory muscles. Jpn J Physiol. 2003;53:243-6.

11. Braun NM, Arora NS, Rochester DF. Force-length relationship of the normal human diaphragm. J Appl Physiol. 1982;53:405-12.

12. De Troyer A, Kirkwood PA, Wilson TA. Respiratory action of the intercostal muscles. Physiol Rev. 2005;85:717-56.

13. De Troyer A, Legrand A, Gevenois PA, Wilson TA. Mechanical advantage of the human parasternal intercostal and triangularis sterni muscles. J Physiol. 1998;513 (Pt 3):915-25.

14. De Troyer A, Legrand A, Wilson TA. Respiratory mechanical advantage of the canine external and internal intercostal muscles. J Physiol. 1999;518 (Pt 1):2839.

15. Proske U, Stuart GJ. The initial burst of impulses in responses of toad muscle spindles during stretch. J Physiol. 1985;368:1-17.

16. Sharp JT. The respiratory muscles in chronic obstructive pulmonary disease. Am Rev Respir Dis. 1986;134:1089-91.

17. Sharp JT, Goldberg NB, Druz WS, Fishman HC, Danon J. Thoracoabdominal motion in chronic obstructive pulmonary disease. Am Rev Respir Dis. 1977;115:47-56.

18. Clarenbach CF, Senn O, Brack T, Kohler M, Bloch KE. Monitoring of ventilation during exercise by a portable respiratory inductive plethysmograph. Chest. 2005;128:1282-90.

19. Konno K, Mead J. Measurement of the separate volume changes of rib cage and 


\section{IZUMIZAKI et al.}

abdomen during breathing. J Appl Physiol. 1967:22:407-22

20. Wolfson MR, Greenspan JS, Deoras KS, Allen JL, Shaffer TH. Effect of position on the mechanical interaction between the rib cage and abdomen in preterm infants. J Appl Physiol. 1992;72:1032-8.

21. Werchowski JL, Sanders MH, Costantino JP, Sciurba FC, Rogers RM. Inductance plethysmography measurement of CPAP-induced changes in endexpiratory lung volume. J Appl Physiol. 1990;68:1732-8.

22. De Troyer A. Effect of hyperinflation on the diaphragm. Eur Respir J. 1997;10:708-13.

23. Russell JA, Bishop B. Vagal afferents essential for abdominal muscle activity during lung inflation in cats. J Appl Physiol. 1976;41:310-5.

24. Road JD, Leevers AM. Inspiratory and expiratory muscle function during continuous positive airway pressure in dogs. J Appl Physiol. 1990;68:1092-100

25. Gorini M, landelli I, Misuri G, Bertoli F, Filippelli M, Mancini M, Duranti R, Gigliotti $\mathrm{F}$, Scano G. Chest wall hyperinflation during acute bronchoconstriction in asthma. Am J Respir Crit Care Med. 1999;160:808-16.

26. Gorini M, Misuri G, Duranti R, landelli I, Mancini M, Scano G. Abdominal muscle recruitment and PEEPi during bronchoconstriction in chronic obstructive pulmonary disease. Thorax. 1997;52:355-61.

27. Ward ME, Ward JW, Macklem PT. Analysis of human chest wall motion using a two-compartment rib cage model. J Appl Physiol. 1992;72:1338-47.

28. Aliverti A, Cala SJ, Duranti R, Ferrigno G, Kenyon CM, Pedotti A, Scano G, Sliwinski P, Macklem PT, Yan S. Human respiratory muscle actions and control during exercise. J Appl Physiol. 1997;83:1256-69.

29. O'Donnell DE, Revill SM, Webb KA. Dynamic hyperinflation and exercise intolerance in chronic obstructive pulmonary disease. Am J Respir Crit Care Med. 2001;164:770-7.

30. O'Donnell DE, Hamilton AL, Webb KA. Sensory-mechanical relationships during high-intensity, constant-work-rate exercise in COPD. J Appl Physiol. 2006;101:1025-35. 